\title{
La enseñanza de educación socioemocional en la escuela primaria desde la perspectiva de la socioformación
}

\author{
The teaching of Socio-Emotional Education in primary school \\ from the perspective of socioformation
}

(iD) Florentino López Piza

Centro Universitario, CIFE

Correo: mercurius_60@hotmail.com

Resumen: Actualmente se enfrentan diversas tensiones conductuales, emocionales y sociales que influyen de manera negativa en el devenir armónico de la humanidad. En este contexto, la educación enfrenta nuevos retos y desafíos que no sólo estarán permeados por la enseñanza de aprendizajes cognitivos, sino por otros aprendizajes emocionales que promuevan la formación integral del ser humano como se establece en los principios filosóficos de la educación mexicana. La presente investigación, tiene como propósito realizar un análisis teórico a partir de la revisión bibliográfica sobre las emociones y la educación socioemocional como área formativa que pretende favorecer el desarrollo armónico e integral de los estudiantes. Se hace un análisis conceptual de las emociones, la educación socioemocional y la socioformación como enfoque emergente de enseñanza que orienta la formación del individuo a partir de la resolución de problemas del contexto, el trabajo colaborativo y el desarrollo del talento humano. Los resultados concluyen que los educadores deben potenciar el desarrollo de las habilidades socioemocionales en los estudiantes para favorecer su formación integral y el despliegue de las capacidades de autoconocimiento, autorregulación y autonomía en el marco de la empatía y la colaboración.

Palabras clave: desarrollo integral; educación socioemocional; emociones, socioformación

\begin{abstract}
Currently, there are behavioral, emotional and social tensions that have a negative influence on the harmonious development of humanity. In this context, education faces new challenges that will not only be permeated by the teaching of cognitive learning, but by other emotional learning that promotes the integral formation of the human being as established in the philosophical principles of Mexican education. The purpose of this research is to carry out a theoretical analysis based on the bibliographical review of emotions and socioemotional education as a formative area that intends to favor the harmonic and integral development of students. A conceptual analysis of emotions, socioemotional education and socioformation is made as an emerging approach to teaching that guides the formation of the individual from the resolution of problems of context, collaborative work and the development of human talent. The results conclude that educators should promote the development of socioemotional skills in students to encourage their comprehensive training and the deployment of self-knowledge, self-regulation and autonomy within the framework of empathy and collaboration.
\end{abstract}

Keywords: emotions; integral development; socioformation; socioemotional education

López Piza, F. (2021). La enseñanza de educación socioemocional en la escuela primaria desde la perspectiva de la socioformación. [The teaching of Socio-Emotional Education in primary school from the perspective of socioformation]. Forhum International Journal of Social Sciences and Humanities, 3(4), 149-161. https://doi.org/10.35766/j.forhum2021.04.03.12

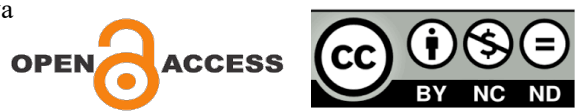




\section{Introducción}

Los procesos de enseñanza-aprendizaje que se gestionan en el aula entre educador y educandos, son mediados por un conjunto de acciones, interacciones y participaciones que se generan con el propósito de construir conocimientos, habilidades, valores y destrezas que se definen en el currículo escolar. En este andamiaje, ha imperado la cultura del saber cognitivo como fin primordial de la acción educativa y se ha dejado de lado las cuestiones emocionales de los estudiantes. Los retos actuales de una sociedad cambiante e inmersa en un sinfín de transformaciones sociales, tecnológicas económicas, políticas, culturales y valorales, demanda de nuevas formas de interacción social, nuevas formas de comunicación y nuevas formas de aprender.

$\mathrm{Al}$ respecto, el sistema educativo mexicano, ha diseñado e implementado una reforma educativa de enfoque humanista que incluye la renovación del mapa curricular, métodos y enfoques de enseñanza que garanticen dar respuesta a las necesidades que plantea una sociedad cambiante y cada vez más exigente con el pleno goce de sus derechos y libertades. Esta reforma fue concretada en el documento Aprendizajes clave para la educación integral, Plan y programas de estudio para la educación básica (SEP, 2017). En la estructura curricular, se introduce por primera vez la asignatura de educación socioemocional, la cual tiene por objetivo desarrollar las habilidades expresivas, de regulación y gestión de las emociones para favorecer la formación integral del educando.

La inclusión de la asignatura de educación socioemocional en el currículo escolar, atiende la necesidad de orientar los procesos de enseñanza-aprendizaje bajo una visión humanista y dialógica de las interacciones humanas, donde se aborden desde una perspectiva formativa los aspectos cognitivos, emocionales y éticos para formar alumnos capaces de conducirse de manera afectiva, autorregulada, autónoma y pacífica en la sociedad; desafíos que hacen falta consolidarse para que los sujetos construyan una actitud crítica, reflexiva, solidaria, empática y convivan en armonía en una sociedad con valores.

En este sentido, el presente artículo tiene como objetivo realizar un análisis conceptual y curricular de la educación socioemocional atendiendo el enfoque del Modelo Educativo de 2017 y de la socioformación como planteamiento formativo que privilegia el desarrollo integral del ser humano a partir de la resolución de problemas del contexto, el trabajo colaborativo, la construcción del proyecto ético de vida y la metacognición como elementos esenciales del aprendizaje (Tobón et al. 2015).

Atendiendo el propósito de la investigación, se establecen las siguientes metas: 1) Analizar la conceptualización de las emociones y la educación socioemocional como proceso de aprendizaje que tiene por objetivo desarrollar las habilidades emocionales de los estudiantes para favorecer su desarrollo integral; 2) Comparar el enfoque didáctico del Modelo Educativo con las aportaciones de la socioformación sobre el desarrollo de las habilidades socioemocionales en los estudiantes; y 3 ) Plantear algunas estrategias de enseñanza que promuevan el desarrollo integral de los estudiantes.

\section{Metodología}

Tipo de estudio

La investigación está basada en la metodología del análisis documental bajo el paradigma cualitativo. El análisis documental consiste en la búsqueda, recuperación, análisis, crítica e interpretación de datos registrados en diversas fuentes documentales: impresas, audiovisuales o 
electrónicas (Ortega et al. 2015); se da una lectura inicial a los documentos y se establecen las categorías de análisis considerando el problema de investigación. Los documentos se analizan a partir de categorías que son los ejes claves para abordar el problema; la información analizada se organiza críticamente respecto al problema de investigación; se establecen posibles vacíos en el conocimiento $\mathrm{y}$ se proponen acciones para futuras investigaciones.

\section{Criterios de Selección de los Documentos}

La investigación documental se inició con la exploración y selección de documentos relativos a las palabras clave que son: emociones, educación socioemocional, desarrollo integral y socioformación. Se buscaron artículos científicos en revistas indexadas y libros en la base de datos de Google académico, Scopus, Web of Science y Elsevier, editados dentro del periodo 2015-2020. Los documentos analizados permitieron recopilar información fidedigna y actualizada sobre el área de estudio, mismos que posibilitaron la exposición y argumentación del tema de investigación (Tabla $1)$.

\section{Tabla 1. Documentos revisados sobre las emociones y educación socioemocional}

\begin{tabular}{|c|c|c|c|}
\hline Registro & Tipo de documento & País & Referencia \\
\hline 1. & Artículo en revista Indexada & México & Bolaños (2020) \\
\hline 2. & Libro & España & Delors (1996) \\
\hline 3. & Artículo en revista Indexada & México & Glejzer et al. (2019) \\
\hline 4. & Libro & España & Goleman (1996) \\
\hline 5. & Artículo de revista indexada & México & Hernández-Mosqueda et al. (2014) \\
\hline 6. & Artículo de revista indexada & Cuba & Laguado et al. (2016) \\
\hline 7. & Artículo de revista indexada & México & McConnell (2019) \\
\hline 8. & Libro & Uruguay & Muslera (2016) \\
\hline 9. & Artículo científico & México & Ortega-Carbajal et al. (2015) \\
\hline 10. & Artículo científico & México & Ortega- Estrada (2017) \\
\hline 11. & Artículo científico & México & Pank \& Acosta (2019) \\
\hline 12. & Artículo científico & Colombia & Rendón (2015) \\
\hline 13. & Artículo de revista indexada & Venezuela & Rojas et al. (2017) \\
\hline 14. & Libro & México & SEP (2017) \\
\hline 15. & Libro & México & SEP (2018) \\
\hline 16. & Libro & España & Soler et al. (2016) \\
\hline 17. & Medio electrónico & México & Tobón (2018) \\
\hline 18. & Documento electrónico & México & Tobón (2013) \\
\hline 19. & Libro & EUA & Tobón (2017) \\
\hline 20. & Libro & México & Tobón (2014) \\
\hline 21. & Artículo de revista indexada & México & Tobón et al. (2015) \\
\hline 22. & Artículo de revista indexada & México & Tobón et al. (2015) \\
\hline
\end{tabular}

Fuente: elaboración propia (2020). 


\section{Categorías de análisis}

Con el propósito de organizar la información para su análisis documental respectivo, se establecieron las siguientes categorías que buscan representar de forma unificada la teoría para facilitar su recuperación, extracción y jerarquización que refleje el tratamiento objetivo de la información (Tabla 2).

\section{Tabla 2. Categorías de análisis}

1. Las emociones.

2. Educación socioemocional en el currículum escolar.

3. La educación socioemocional desde la perspectiva de la socioformación.

4. Estrategias didácticas para el desarrollo de habilidades socioemocionales.

5. El papel del maestro en la enseñanza de la educación socioemocional.

Fuente: Elaboración propia (2020).

\section{Resultados}

Las emociones

Una de las principales finalidades de la educación en México es lograr el pleno desarrollo integral del ser humano, formarlo con una nueva visión de una ciudadanía libre, democrática y con pleno goce de sus derechos y facultades. En este proceso, se observan como mínimo dos aspectos fundamentales que deben caracterizar la construcción de la personalidad: el desarrollo cognitivo y el desarrollo emocional. Paradójicamente, la escuela se ha centrado en privilegiar el aspecto cognitivo antes que el desarrollo emocional de los educandos, lo que ha implicado que se observen altos índices de reprobación y rezago escolar, dificultades de aprendizaje, estrés ante la resolución de exámenes y diversos problemas de disciplina que inciden en estados emocionales negativos (Soler et al. 2016).

Ante estas situaciones, resulta imperativo realizar un análisis reflexivo sobre el papel que juegan las emociones en la construcción social del conocimiento y en las interacciones sociales que enfrentan los seres humanos en la cotidianidad. Las emociones son expresiones de carácter biológico y cognitivo que cobran sentido en ambientes sociales. Se pueden caracterizar como positivas cuando generan sentimientos placenteros y significa que la situación es de beneficio como la felicidad y el amor; negativas cuando se acompañan con sentimientos desagradables y se percibe una situación de amenaza como el miedo, la ansiedad, la ira, la tristeza, o neutras cuando no expresan ningún sentimiento como la esperanza y la sorpresa (Muslera, 2016).

La importancia del control y regulación de las emociones surge de la necesidad que tiene el ser humano de no dejarse llevar por los impulsos emocionales, porque esto tendría consecuencias negativas en el ámbito personal y social. El desarrollo y comprensión de las emociones en los niños, es un proceso gradual y permanente del aprendizaje que les permite ir cambiando sus estrategias para poder enfrentar las distintas experiencias a través del control de las emociones y del conocimiento de sus causas (Glejzer et al. 2019). El control y regulación de las emociones generan estados de bienestar personal y estabilidad emocional que pueden favorecer los procesos de autoconocimiento, autoconfianza y toma de decisiones de forma asertiva.

La literatura ha tratado de demostrar que las emociones tienen ciertos efectos sobre los procesos mentales de las personas, al respecto, McConnell (2019), plantea que las emociones pueden afectar la percepción, atención, memoria y el razonamiento; en contraste, describe que las emociones 
positivas integrales como el gusto, la motivación y el interés por aprender, pueden influenciar en gran medida los procesos de aprendizaje de los alumnos, facilitando el uso de las estrategias de aprendizaje profundo y pensamiento creativo para alcanzar sus metas.

Goleman (1996) definió la inteligencia emocional como la capacidad para reconocer nuestros propios sentimientos y de los demás, de motivarnos y de manejar asertivamente nuestras emociones y relaciones sociales. Dicho autor sostenie que, personas con bajo nivel de coeficiente intelectual (CI), pero que poseen una alta capacidad para regular sus emociones, son capaces de superar a aquellos individuos que tienen un alto CI, pero un bajo nivel de manejo de las emociones. Este contraste, refleja que las emociones juegan un papel determinante en el desarrollo integral de la personalidad y, en consecuencia, el sistema educativo estaba obligado a incluir en el currículo escolar el diseño de un cuerpo de contenidos enfocados a atender el desarrollo de las competencias emocionales de los educandos para que adquieran habilidades de autorregulación, empatía y gestión asertiva de las emociones. De esta forma, se plantea la posibilidad de que, en los procesos educativos, se incluya un conjunto de contenidos orientados a favorecer en los educandos el desarrollo de habilidades emocionales.

El Informe de la Organización de las Naciones Unidas para la Educación, la Ciencia y la Cultura (UNESCO) de la Comisión Internacional sobre la Educación para el siglo XXI, La Educación encierra un tesoro (Delors, 1996) establece que para hacer frente a los numerosos desafíos del porvenir, se hace imprescindible asignar nuevos objetivos a la educación, modificar el enfoque de prevalencia cognitiva y articular una nueva visión sustentada en la observancia de formar al educando en torno a los cuatro pilares básicos: 1) aprender a conocer; 2) aprende a hacer; 3) aprender a ser; y 4) aprender a vivir juntos. La educación tiene que adaptarse a los nuevos cambios y exigencias que plantea la nueva sociedad del conocimiento y de la información, convertirse en un instrumento de vanguardia para que la humanidad alcance los ideales de paz, armonía, libertad y justicia social, forjando al educando bajo un enfoque holista e integral.

\section{Educación socioemocional en el currículo escolar}

En el sistema educativo mexicano, a partir de la puesta en práctica del plan de estudios 2011, se incluyeron las competencias socioemocionales en el campo formativo: desarrollo personal y para la convivencia, el cual tiene como finalidad que los estudiantes aprendan a actuar con juicio crítico a favor de la democracia, la libertad, la paz, el respeto y la legalidad; también estipula el manejo armónico de las relaciones interpersonales y afectivas, la autonomía y la toma de decisiones.

A partir de la Reforma Educativa que se concretó en los Aprendizajes Clave para la Educación Integral (SEP, 2017), se incluyó en el área de desarrollo personal y social, la enseñanza de la educación socioemocional, la cual es concebida como un proceso de aprendizaje por medio del cual los educandos integran en su persona conceptos, valores, actitudes y habilidades que les permiten comprender y manejar sus emociones, construir su identidad personal, mostrar atención y cuidado hacia los demás, colaborar, establecer relaciones positivas, tomar decisiones responsables y aprender a manejar situaciones retadoras de manera constructiva y ética.

El propósito que se establece es lograr que los estudiantes desarrollen y pongan en práctica herramientas para generar un sentido de bienestar consigo mismo y hacia los demás, a través de experiencias, prácticas y rutinas vinculadas a las actividades escolares; que comprendan y aprendan a regular de forma positiva sus emociones impulsivas o aflictivas y que logren que su vida emocional $\mathrm{y}$ relaciones interpersonales sean un factor de motivación y aprendizaje para lograr metas 
constructivas en la vida (SEP, 2017). Para ello, es necesario que la educación impulse la internalización de los valores humanistas como el respeto a la vida y la dignidad humana, la igualdad de derechos y la justicia social, la diversidad cultural y social, así como la solidaridad y la corresponsabilidad en la construcción de un mundo mejor.

La educación socioemocional puede contribuir a que los estudiantes logren sus metas, se relacionen de manera sana y pacífica en la escuela, en la familia y en la comunidad; reconozcan y valoren la diversidad sociocultural y la importancia de la inclusión como medio para garantizar la igualdad, la equidad, la justicia y la paz social. Los propósitos que se persiguen desarrollar para el nivel de educación primaria, incluyen las habilidades esenciales para que los alumnos aprendan a reconocer y regular sus emociones, trabajar de manera colaborativa y emprender proyectos que promuevan la participación social y el desarrollo de valores morales, sociales y ambientales (ver tabla $3)$.

Tabla 3. Propósitos de la educación socioemocional para educación primaria.

1. Desarrollar habilidades y estrategias para la expresión, regulación y la gestión de las emociones; el conocimiento de las causas y efectos de expresión emocional; la tolerancia a la frustración y la templanza para postergar las competencias inmediatas.

2. Desarrollar formas de comunicación asertiva y escucha activa.

3. Reconocer y valorar la diversidad cultural y el medioambiente, y la importancia de la inclusión como mecanismo para garantizar la igualdad y la paz social.

4. Reafirmar el ejercicio de la autonomía a través de la participación en acciones y proyectos colectivos que busquen el beneficio de la comunidad.

5. Fortalecer la autoestima para conducirse con seguridad, respeto y sentido de agencia, que es la capacidad para llevar a cabo acciones que generen mayores oportunidades de vida para sí mismo y para los demás.

6. Reconocer el poder de la empatía para establecer relaciones sinceras, respetuosas y duraderas. Fuente: Aprendizajes clave para la educación integral (SEP, 2017).

La propuesta educativa plantea aportar a los estudiantes y docentes herramientas y estrategias para trabajar el ámbito instruccional y las interacciones que se desarrollan cotidianamente en el aula, en aspectos socioemocionales esenciales para favorecer el aprendizaje y la convivencia escolar. El enfoque de enseñanza busca orientar la práctica pedagógica para favorecer el desarrollo integral de los educandos y alcanzar los propósitos educativos que van más allá de los conceptos disciplinares y académicos a partir de actuaciones encaminadas a incorporar en los procesos de enseñanza, el desarrollo de las competencias socioemocionales.

El plan curricular asigna 30 minutos de trabajo lectivo a la semana en educación primaria y se consideran 25 indicadores de logro para cada grado, los cuales deben ser considerados en la planeación didáctica para trabajar cada una de las dimensiones socioemocionales en igual número de sesiones (SEP, 2017). La organización curricular se estructura con base a cinco dimensiones, las cuales agrupan un conjunto de habilidades que guían las interacciones educativas enmarcadas en los cuatro pilares de la educación: aprender a aprender, aprender a ser, aprender a hacer y aprender a convivir (tabla 4). 
Tabla 4. Organizadores curriculares.

Dimensiones Habilidades

\begin{tabular}{lll} 
1. Autoconocimiento & $\begin{array}{l}\text { Atención, conciencia de las propias emociones, autoestima, aprecio y } \\
\text { gratitud, bienestar. } \\
\text { Metacognición, expresión de las emociones, regulación de las } \\
\text { emociones, autogeneración de emociones para el bienestar y } \\
\text { perseverancia. }\end{array}$ \\
3. Autorregulación & $\begin{array}{l}\text { Iniciativa personal, identificación de necesidades y búsqueda de } \\
\text { emociones, liderazgo y apertura, toma de decisiones y compromisos, } \\
\text { autoeficacia. }\end{array}$ \\
4. Empatía & $\begin{array}{l}\text { Bienestar y trato digno hacia otras personas, toma de perspectiva en } \\
\text { situaciones de desacuerdo o conflicto, reconocimiento de prejuicios } \\
\text { asociados a la diversidad, sensibilidad hacia personas y grupos que } \\
\text { sufren exclusión o discriminación, cuidado de otros seres vivos y la } \\
\text { naturaleza. } \\
\text { Comunicación asertiva, responsabilidad, inclusión, resolución de } \\
\text { conflictos e interdependencia. }\end{array}$ \\
\hline
\end{tabular}

Fuente: Aprendizajes clave para la educación integral (SEP, 2017).

Como puede observarse, cada una de las dimensiones de la educación socioemocional, tienen un conjunto de habilidades específicas que tienen diferentes indicadores de logro para cada grado escolar, mismos que señalan algunas conductas y actitudes que los alumnos pueden lograr como resultado de haber desarrollado cada una de ellas. Se comprende que el desarrollo de las habilidades y competencias socioemocionales, favorecen la formación integral de los estudiantes y que los aprendizajes adquieren sentido cuando verdaderamente contribuyen al pleno desarrollo personal y social de los individuos, considerando como ejes primordiales las competencias clave de aprender a aprender y aprender a convivir (Ortega, 2017).

\section{La educación socioemocional desde la perspectiva de la socioformación}

La socioformación como un enfoque formativo emergente que ha tenido gran relevancia en la educación, se ha definido como una perspectiva educativa que orienta la formación integral del individuo a partir del abordaje de problemas del contexto, en el marco del trabajo colaborativo, atendiendo el proyecto ético de vida de las personas, el emprendimiento mediante proyectos transversales, la gestión y co-creación del conocimiento y la metacognición, considerando como base primordial las tecnologías de la información y comunicación (Tobón et al. 2015).

La socioformación privilegia una educación centrada en la formación integral de las personas, del desarrollo del talento humano, emprendimiento y de la colaboración como estrategias ineludibles para favorecer las competencias cognitivas, afectivas e integrales que potencien las relaciones interpersonales y sociales en un ambiente de mejora de la calidad de vida, el tejido social, la convivencia y la inclusión (Tobón, 2017). Sin embargo, hay una clara ambigüedad sobre la conceptualización de las habilidades socioemocionales y la forma en que deben ser abordarlas en el aula para favorecer en los alumnos el desarrollo de las competencias necesarias para afrontar los retos del contexto social y el desarrollo integral. 
Al respecto, Tobón (2018), define a las habilidades socioemocionales desde la socioformación como actuaciones integrales en torno al manejo de las emociones para alcanzar la realización personal, promover el desarrollo social y asegurar la sostenibilidad ambiental de manera integrada con base a la resolución de problemas y el afrontamiento de los retos de la vida mediante la ética, la creatividad y el mejoramiento continuo. Estas habilidades forman parte del proyecto ético de vida, el cual consiste en trabajar por el bienestar propio y de los demás, poniendo en práctica los valores universales como la responsabilidad, el respeto, la honorabilidad, la equidad, la solidaridad, la humildad y la promoción de la vida.

\section{Estrategias didácticas para el desarrollo de habilidades socioemocionales}

La propuesta metodológica desde la socioformación para abordar el desarrollo de las habilidades socioemocionales, parte de la realización de proyectos formativos transversales con las demás áreas o asignaturas del currículo escolar. De esta forma, se promueve la formación mediante la identificación, comprensión, argumentación y resolución de problemas del contexto que activen las emociones de los estudiantes; además, se proponen otras estrategias como la autoevaluación, coevaluación, metacognición, meditación y relajación; juegos de reto y coaching, como instrumentos y procesos de reflexión y actuación que aborden lo afectivo-emocional, el bienestar psicológico, lo procedimental y las acciones en el ámbito social y ambiental (Tobón, 2018). La realización de los proyectos formativos que contemplen los aspectos cognitivos y emocionales, así como las acciones de reflexión y valoración sobre el desarrollo integral del talento humano, darán cuenta de la realización personal y del logro de las habilidades socioemocionales de los estudiantes.

Los proyectos formativos son una estrategia de enseñanza que la socioformación ha adaptado para mediar el desarrollo del talento humano impulsando la formación de las personas a partir de la resolución de problemas del contexto bajo el enfoque del trabajo colaborativo; se conceptualizan como un conjunto de actividades articuladas para identificar, interpretar, argumentar y resolver problemas mediante la colaboración y la presentación de evidencias de desempeño que reflejen la mejora de la situación que abordan, buscando la formación de estudiantes competentes, emprendedores y éticos (Tobón, 2014). Este tipo de proyectos tienen el propósito de favorecer en los alumnos la construcción de su propio aprendizaje a partir de del desarrollo de proyectos reales del contexto, la comunicación asertiva, el trabajo colaborativo y el empleo de las tecnologías de la información y comunicación (Laguado y Villamizar, 2016).

La realización de los proyectos formativos, debe favorecer también el fortalecimiento del proyecto ético de vida de los estudiantes, promoviendo la consolidación de valores como la responsabilidad, la honestidad, la solidaridad, la equidad, el compromiso y el respeto; orientando la realización personal al mismo tiempo que se contribuye a la mejora de la calidad de vida, el bienestar social, la convivencia y el desarrollo sustentable (Tobón, Cardona et al. 2015; Luna-Nemecio et al, 2020). Los proyectos formativos como alternativa de enseñanza participativa, colaborativa e interactiva que parten de la resolución de problemas concretos del contexto y la gestión del conocimiento para dar respuesta a las demandas ambientales, culturales y sociales, promueven la formación en valores y el desarrollo socioemocional de los estudiantes.

El Trabajo colaborativo en este contexto, se entiende como un proceso a través del cual las personas trabajan con otras mediante actividades articuladas para lograr una meta común, lo cual implica comunicarse con asertividad, unir fortalezas y solucionar los conflictos que se presenten. En el ámbito escolar, el trabajo colaborativo se promueve entre los estudiantes, buscando la participación 
de todos y la articulación de acciones para interpretar, argumentar y resolver problemas a partir de diferentes modalidades de trabajo: a) todos trabajan un mismo proyecto; b) los alumnos se organizan en equipos y cada equipo aborda un problema del proyecto general; y c) cada estudiante trabaja un proyecto, pero durante el proceso se comparten los avances con los pares, promoviendo la participación de todos y al orientación del docente (Tobón, González et al. 2015).

Otra de las estrategias de enseñanza que pueden favorecer el desarrollo de las habilidades socioemocionales en los estudiantes, es la técnica del sociodrama, donde se pueden analizar situaciones de la vida real a partir de la actuación. El sociodrama es la representación de un hecho o situación de la vida real en un espacio determinado y con la presencia y participación de los mismos alumnos, quienes posteriormente a la problemática representada, analizan la situación a mayor profundidad (Tobón, 2013). Esta técnica, permite representar cualquier tema que sucede en la vida cotidiana y escolar, situaciones problemáticas, actuaciones contradictorias e ideas encontradas que pueden favorecer el análisis de hechos sociales y generar espacios de discusión para dar solución a los problemas, el desarrollo de habilidades interpersonales, el trabajo en equipo y colaborativo, profundizar sus conocimientos sobre las temáticas abordadas y la toma de decisiones.

\section{El papel del maestro en la enseñanza de la educación socioemocional}

La propuesta de enseñanza de la educación socioemocional que se plantea en los aprendizajes clave para la educación integral (SEP, 2017), alude a la figura del docente como un agente mediador y facilitador del aprendizaje, promoviendo el diálogo, el respeto y la empatía como principales estrategias de trabajo. En este marco, debe privilegiarse que se generen espacios de escucha activa entre alumnos y docentes en un ambiente de respeto, que promuevan la participación y apropiación de conceptos, actitudes y pautas de convivencia que caracterizan a las dimensiones socioemocionales descritas.

Entre las estrategias pedagógicas de aprendizaje que se proponen para promover el desarrollo de las habilidades socioemocionales, destacan: a) aprendizaje situado, como enfoque de enseñanza donde los alumnos aprenden en un contexto pertinente y se involucre la afectividad, el respeto y la participación; b) aprendizaje dialógico, donde se favorezcan los procesos de autoconocimiento, autorregulación, autonomía, la colaboración y resolución pacífica de conflictos; c) aprendizaje basado en el juego, como generador del desarrollo de la imaginación y la creatividad; d) aprendizaje basado en proyectos, como estrategia que genera oportunidades de autoconocimiento, autonomía, autorregulación y toma de decisiones, y e) aprendizaje basado en método de casos, como estrategia que brinda espacios para poner en práctica el análisis de experiencias y situaciones problemáticas de la vida real para que a partir de la autorregulación, autonomía y la toma de decisiones, se generen soluciones pacíficas (SEP, 2017).

Lograr un pleno desarrollo de las habilidades o competencias socioemocionales en los educandos, requiere que el docente tome como punto de partida el clima del aula, promueva un ambiente de respeto y confianza, genere un sentido de pertenencia, construya relaciones basadas en la empatía que den lugar a la participación y apropiación de actitudes, conceptos y pautas de convivencia que definan el acto de aprender como un proceso que refleja niveles de conciencia y desarrollo humano para lo afectivo, lo social, lo espiritual y lo físico, trascendiendo lo puramente cognitivo y memorístico (SEP, 2018). La educación socioemocional debe abordarse bajo un enfoque transversal como un proceso interdisciplinario articulado con las demás asignaturas del currículo y 
en observancia a un contexto intercultural como un principio de vida que promueve el intercambio y el respeto a las relaciones interpersonales y colectivas en un ambiente de equidad.

En este contexto, el enfoque de la socioformación es congruente con el planteamiento y exigencias actuales del sistema educativo para favorecer en los estudiantes las competencias socioemocionales que les permitan construir su proyecto ético de vida, desarrollar su talento personal y social (Tobón \& Luna-Nemecio, 2020), fortalecer el trabajo colaborativo y lograr su pleno desarrollo integral. Un proyecto ético de vida implica buscar la realización personal, mejorar la calidad de vida, desarrollar el talento a través de la resolución de problemas contextuales, la creatividad, los valores universales y fortalecer el autocuidado de la salud y protección de la vida (Tobón, 2017). En este tenor, se requiere que los educadores en primer término, desarrollen sus propias competencias, habilidades y capacidades sociales y emocionales, dejando de lado la improvisación (Pank y Acosta, 2019), para diseñar planificaciones y estrategias didácticas que promuevan la construcción social del conocimiento y el desarrollo armónico e integral del educando.

\section{Discusión y conclusiones}

El análisis bibliográfico refleja congruencia en la necesidad de promover en los estudiantes el desarrollo de las habilidades socioemocionales orientadas a favorecer su desarrollo armónico e integral. Paradójicamente, las prácticas educativas que gestionan los educadores en las aulas, expresan la falta de estrategias y técnicas didácticas que promuevan la construcción de dichas habilidades formativas. Ante la emergencia de impulsar acciones encaminadas a disminuir los conflictos sociales y promover una educación centrada en la formación en valores; las políticas educativas han incluido en el currículo escolar la enseñanza de la educación socioemocional como área formativa que promueva en los alumnos el desarrollo de las habilidades socioemocionales y favorezcan la construcción de competencias autogestivas, cognitivas, actitudinales y conductuales (Uribe, 2015). En este propósito, se plantean estrategias de aprendizaje dialógicas, situadas y lúdicas, basadas en la realización de proyectos y de análisis de casos para favorecer las capacidades de autoconocimiento, autorregulación, autonomía, la colaboración y toma de decisiones para resolver conflictos de manera pacífica y en el marco del respeto a los derechos humanos (SEP, 2017).

Los proyectos formativos son otra estrategia que plantea el enfoque de la socioformación como paradigma emergente de enseñanza que se centra en el desarrollo del talento humano a partir de la resolución de problemas del contexto y el trabajo colaborativo (Bermeo-Yaffar \& Luna-Nemecio, 2020). Se propone el diseño de proyectos formativos transversales que articulen actividades de aprendizaje con otras asignaturas del currículo que promuevan la participación activa de los estudiantes en la resolución de problemas reales del contexto, basados en la colaboración, la gestión del conocimiento, el emprendimiento y el desarrollo de habilidades socioemocionales (Tobón, 2015). En este ámbito, se requiere que los docentes pongan en práctica sus competencias cognitivas, pedagógicas y didácticas para generar espacios de diálogo, de participación, de acuerdos, de colaboración y acompañamiento en el diseño, desarrollo y evaluación de los proyectos formativos. Además, se debe fortalecer en los estudiantes la comunicación asertiva, el liderazgo y trabajo en equipo, gestión de proyectos educativos, mediación de la formación integral, evaluación de competencias y empleo de las tecnologías de la información y comunicación (Laguado, 2016).

Los modelos y paradigmas educativos, tradicionalmente se han centrado en el desarrollo cognitivo de los estudiantes, dejando en segundo término la atención y desarrollo de sus emociones 
como fenómenos de carácter biológico-cogntivo que pueden expresarse en ambientes sociales. En los años recientes, derivado de diversos conflictos sociales, falta de valores y la transgresión constante de los derechos humanos, los sistemas educativos han incluido en los currículos escolares la enseñanza de la educación socioemocional como una alternativa enfocada a lograr el desarrollo integral del ser humano. La educación socioemocional en este sentido debe asumirse como un área formativa que de respuesta puntual a la demanda que reclama la formación de un ciudadano que sea capaz de vivir en armonía consigo mismo y con los demás a través del fortalecimiento de las relaciones interpersonales, la independencia, la disciplina, la responsabilidad, la alegría de vivir y la actitud positiva (Rojas et al. 2017).

El aprendizaje social y emocional debe ser considerado como un proceso de desarrollo de competencias y habilidades básicas en los alumnos para que enfrenten con éxito los desafíos que depara la sociedad del conocimiento, las interacciones sociales y los problemas ambientales. Sin embargo, a pesar de que la educación socioemocional se ha incluido en el currículo escolar, hay evidencias de la ausencia de herramientas y estrategias metodológicas que los docentes deben emplear para trabajar de manera efectiva el ámbito socioemocional de los estudiantes (Rojas et al. 2017).

Uno de los paradigmas que se ha planteado desde la socioformación para dar respuesta a los retos y desafíos que enfrenta la educación, es la docencia socioformativa, entendida como la concatenación de las acciones que emprende el docente mediador para lograr la formación integral y el desarrollo de competencias en los alumnos con base en ambientes de aprendizaje centrado en la resolución de problemas (Hernández et al. 2014). La propuesta metodológica incluye la realización de proyectos formativos, resolución de problemas del contexto, juego de retos y coaching creativo, como estrategias que favorecen la participación, la reflexión, el trabajo colaborativo, el desarrollo del talento humano y la realización personal (Tobón, 2018).

Lograr que los alumnos se formen como entes integrales, resulta crucial que desde la ética docente, se asuma una conciencia social y educativa crítica para impulsar la construcción y expresión de valores mediante la participación activa y corresponsable con la comunidad escolar, diseñando situaciones de aprendizaje que impulsen la realización personal, la construcción del proyecto ético de vida, el trabajo colaborativo y el desarrollo sostenible (Bolaños, 2020). Esto implica que los educadores como agentes mediadores de la enseñanza, deben tener conciencia de sus propias emociones, motivación, empatía, innovación y ser competentes en el manejo de habilidades cognitivas y sociales para promover ambientes de aprendizaje participativos, democráticos e incluyentes.

Atendiendo esta necesidad, se hace imprescindible generar espacios de actualización y capacitación docente sobre las estrategias de enseñanza que favorezcan el desarrollo de las habilidades socioemocionales de los estudiantes, la formación en valores y la construcción de un proyecto ético de vida para la realización personal (Tobón, 2017).

\section{Referencias}

Bermeo-Yaffar, F. \& Luna-Nemecio, J. (2020). Socioformación y pensamiento matemático. Cartografía conceptual sobre el aprendizaje por proyectos. Política y Cultura, (54), 215-233. https://polcul.xoc.uam.mx/index.php/polcul/article/view/1443

Bolaños Arias, G. (2020). La formación en valores ante la crisis del COVID-19: retos para la Educación Media Superior en México. [Formation of values in the light of COVI-19 crisis: 
challenges for the High School Education in México]. Forhum International Journal of Social Sciences and Humanities, 2(3), 22-33. https://doi.org/10.35766/jf20233

Delors, J. (1996). "Los cuatro pilares de la educación" en La educación encierra un tesoro. Informe a la UNESCO de la Comisión internacional sobre la educación para el siglo XXI. Santillana/UNESCO. pp. 91-103.

Glejzer, C., Ciccarelli, A., Chomnalez, M., \&Ricci, A. (2019). La incidencia de las emociones sobre los procesos de aprendizaje en niños, niñas y jóvenes en contextos de vulnerabilidad social. Voces De La Educación, 2, 113-128.

https://www.revista.vocesdelaeducacion.com.mx/index.php/voces/article/view/215

Goleman, D. (1996). La Inteligencia Emocional. Kairós.

Hernandez Mosqueda, J., Tobón, S. \& Vázquez Antonio, J. (2014). Estudio conceptual de la docencia socioformativa. Ra Ximhai, 9, 89-101.

http://dx.doi.org/10.35197/rx.10.03.e1.2014.06.jh

Laguado Jaimes, E. \& Villamizar Osorio, M. (2016). Desarrollo de la estrategia pedagógica "Proyectos Formativos" para fomentar la integralidad del conocimiento disciplinar. Revista cubana de enfermeria, 32 (4), 126-135. https:/www.medigraphic.com/pdfs/revcubenf/cnf2016/cnf164j.pdf

Luna-Nemecio, J., Tobón, S., \& Juárez, H. (2020) Sustainability-based on socioformation and complex thought or Sustainable Social Development. Resources, Environment and Sustainability, 2, 1-8. https://doi.org/10.1016/j.resenv.2020.100007

McConnell, M. Emociones en educación: cómo las emociones, cognición y motivación influyen en el aprendizaje y logro de los estudiantes. Revista Mexicana de Bachillerato a Distancia, 11 (21), 109-129. http://dx.doi.org/10.22201/cuaed.20074751e.2019.21.68217.

Muslera, M. (2016). Educación emocional en niños de 3 a 6 años. Universidad de la República. https://bit.ly/2NOrv2v

Ortega Carbajal, M., Hernández Mosqueda, J., \& Tobón, S. (2015). Análisis documental de la gestión del conocimiento mediante la cartografía conceptual. $R a$ Ximhai, 11(4) ,141-160. http://dx.doi.org/10.35197/rx.11.01.e2.2015.09.mo

Ortega Estrada, F. (2017). Principios e implicaciones del Nuevo Modelo Educativo. Revista Latinoamericana de Estudios Educativos, 47 (1),43-62. https://www.redalyc.org/articulo.oa?id=270/27050422003

Pank Valenzuela, C., \& Acosta Ramos, S. L. (2019). Educación socioemocional: Un pendiente en la formación docente- http://www.conisen.mx/memorias2019/memorias/1/P886.pdf

Rendón Uribe, A. (2015). Educación de la competencia socioemocional y estilos de enseñanza en la educación media. Sophia, 11(2), 237-256.

https://www.redalyc.org/articulo.oa?id=4137/413740778009

Rojas, F., Escalante, D., \& Bermúdez, L. (2017). Competencias socioemocionales de los docentes en formación. Acción pedagógica, 120-129. https://dialnet.unirioja.es/servlet/articulo?codigo=6344979

Secretaría de Educación Pública. (2017). Aprendizajes clave para la educación integral. Plan y programas de estudio para la educación básica. SEP

Secretaría de Educación Pública. (2018). Educación socioemocional y aprendizaje. SEP

Soler, J., Aparicio, L., Díaz, O., Escolano, E., \& Rodríguez, A. (2016). Inteligencia Emocional y Bienestar. Universidad San Jorge. 
Tobón, S. (2018). Cartografía de las habilidades socioemocionales. Una síntesis desde la socioformación. México: Centro Universitario CIFE. https://cife.edu.mx/recursos/2018/12/18/cartografía-de-las-habilidades-socioemocionales.

Tobón S. (2013). Didáctica Vivencial Escénica: el Método de los Sociodramas. Cife. https://issuu.com/cife/docs/ebook_sociodramas_1.0

Tobón, S. (2014). Proyectos formativos: teoría y práctica. Pearson.

Tobón, S. (2017). Ejes esenciales de la sociedad del conocimiento y la socioformación. Kresearch.

Tobon, S., Nambo, J., González, L. \& Vázquez Antonio, J. (2015). La Socioformación: Un Estudio Conceptual. Paradigma, 36 , 7-29. http://ve.scielo.org/scielo.php?script=sci_arttext\&pid=S1011-22512015000100002

Tobon, S., Cardona, S., Ramos, J. \& López, J. (2015). Proyectos formativos y desarrollo del talento humano para la sociedad del conocimiento. Acción Pedagógica. 24 (1), 20-31. https://dialnet.unirioja.es/servlet/articulo?codigo $=6224808$

Tobón, S. \& Luna-Nemecio, J. (2020). Proposal for a new talent concept based on socioformation. Educational Philosophy and Theory 53(1), 21-33. https://doi.org/10.1080/00131857.2020.1725885 